\title{
Insertions of mitochondrial DNA into the nucleus-effects and role in cell evolution
}

\author{
María J. Puertas and Mónica González-Sánchez
}

\begin{abstract}
We review the insertion of mitochondrial DNA (mtDNA) fragments into nuclear DNA (NUMTS) as a general and ongoing process that has occurred many times during genome evolution. Fragments of mtDNA are generated during the lifetime of organisms in both somatic and germinal cells, by the production of reactive oxygen species in the mitochondria. The fragments are inserted into the nucleus during the double-strand breaks repair via the non-homologous end-joining machinery, followed by genomic instability, giving rise to the high variability observed in NUMT patterns among species, populations, or genotypes. Some de novo produced mtDNA insertions show harmful effects, being involved in human diseases, carcinogenesis, and ageing. NUMT generation is a non-stop process overpassing the Mendelian transmission. This parasitic property ensures their survival even against their harmful effects. The accumulation of mtDNA fragments mainly at pericentromeric and subtelomeric regions is important to understand the transmission and integration of NUMTs into the genomes. The possible effect of female meiotic drive for mtDNA insertions at centromeres remains to be studied. In spite of the harmful feature of NUMTs, they are important in cell evolution, representing a major source of genomic variation.
\end{abstract}

Key words: mitochondrial DNA, NUMT, ageing, centromere, parasitic genetic elements.

Résumé : Les auteurs font une revue de la littérature sur le sujet de l'insertion de fragments d'ADN mitochrondrial (mtDNA) au sein de l'ADN nucléaire, ou NUMTS (pour "Nuclear Mitochondrial DNA Segment"), lesquels sont le fruit d'un processus général et continu qui est survenu plusieurs fois au cours de l'évolution des génomes. Les fragments de mtDNA sont générés tout au long de la vie des organismes, à la fois dans les cellules somatiques et germinales, en réponse à la production d'espèces réactives d'oxygène dans la mitochondrie. Les fragments sont insérés dans le noyau lors de la réparation des bris bicaténaires par la machinerie de jonction d'extrémités non-homologues, laquelle insertion est suivie d'une instabilité génomique, ce qui donne naissance à une grande variabilité dans l'occurrence des NUMTS chez les espèces, populations et génotypes. Certaines insertions de novo de NUMTS entraînent des effets néfastes, dont des maladies chez l'humain, la cancérogenèse et le vieillissement. La génération de NUMTS est un processus continuel qui se superpose à la transmission mendélienne. Cette propriété parasitique assure leur survie en dépit d'effets néfastes. L'accumulation de fragments de mtDNA, principalement dans les régions péricentromériques et subtélomériques, est un phénomène important pour comprendre la transmission et l'intégration des NUMTS dans les génomes. Un possible effet de biais de ségrégation ("meiotic drive") pour les insertions centromériques de mtDNA chez les femelles reste à étudier. En dépit des effets néfastes des NUMTS, ils sont importants pour l'évolution de la cellule du fait qu'ils constituent une source importante de variation génomique. [Traduit par la Rédaction]

Mots-clés : ADN mitochondrial, NUMTS, vieillissement, centromère, éléments génétiques parasitaires.

\section{Introduction}

The evolution of the DNA-carrying cell organelles, mitochondria and chloroplasts, may be considered under three related points of view: (i) the evolution from bacteria to organelles, according to the endosymbiotic origin of the eukaryotic cell; (ii) the evolution of organelle chromosomes producing a large variability in size and structure in different species, resulting in the nearly-unidirectional loss of DNA from the former bacterial chromosomes to the extant organelle chromosomes, including the insertion of organelle-coding genes in the main nuclear DNA; and (iii) the phylogenetic relationships among species or pop-

Received 31 August 2019. Accepted 27 April 2020.

M.J. Puertas* and M. González-Sánchez. Departamento de Genética, Fisiología y Microbiología, Facultad de Biología, Universidad Complutense, José Antonio Novais 2, 28040 Madrid, Spain.

Corresponding author: Mónica González-Sánchez (email: mgs@ucm.es).

${ }^{*}$ M.J. Puertas currently serves as an Associate Editor; peer review and editorial decisions regarding this manuscript were handled by Dan Bock.

Copyright remains with the author(s) or their institution(s). Permission for reuse (free in most cases) can be obtained from copyright.com. 
ulations that can be established to compare the DNA sequences of their organelles, as well as the insertions of organelle DNA into nuclear DNA.

In the present work, we review the insertion of mitochondrial DNA (mtDNA) fragments into nuclear DNA (nDNA), considering that the mtDNA transfer to the nucleus seems to be a general evolutionary trend that may have occurred many times along the genome evolution and it is still an ongoing process, including the lifetime of many organisms. This is a type of intracellular horizontal gene transfer whose effects on the individuals undergoing the transfer and the long-term evolutionary consequences are of general biological interest.

The comparison between mitochondrial and bacterial genomes has shown that mitochondria share a common ancestor with the Alphaproteobacteria. The first complete genome sequence of the obligate intracellular parasite Rickettsia prowazekii showed similarities to mitochondrial genes (Andersson et al. 1998). More recently, Thrash et al. (2011) determined a common origin of mitochondria and SAR11 clade as a sister group to the Rickettsiales. These results are accepted as a proof that the symbiotic event that gave origin to mitochondria occurred only once in cell evolution.

The sequencing of mtDNA in a number of animal, plant, fungi, and protists has shown that there is a large variation in structure and DNA amount among mitochondria of different species. Remarkable is the case of Jakobid protists, showing the most bacteria-like mitochondrial genomes in size and genetic structure (Burger et al. 2013). All mitochondrial genomes known are reductions of these protist mitochondrial genomes, which indeed favors the idea of the uniqueness of the symbiotic event giving rise to mitochondria. Despite their large diversity, a common feature shared by all known mitochondria is that a few of their constitutive proteins are coded by the mitochondrial genes, whereas most of the mitochondrial proteins are coded by nuclear genes.

Not all eukaryotes contain mitochondria. Anaerobic eukaryotes, such as some Ciliates, Trichomonads, Amoeboflagellates, and fungi, do not contain mitochondria, but their cell energy is provided by organelles called hydrogenosomes, which produce ATP and hydrogen anaerobically. Other anaerobic or microaerophilic organisms, such as Microsporidia, contain cell organelles called mitosomes. Although the evolutionary origin of both hydrogenosomes and mitosomes is difficult to establish because both have lost their DNA entirely, protein-based phylogenies, particularly the Hsp70 family, show that both are evolutionarily related to mitochondria (Williams et al. 2002; Embley et al. 2003).

All these facts may be interpreted as the evidence of a single symbiotic event giving rise to mitochondria as a cell organelle, and a general evolutionary trend consisting in the gradual loss of mitochondrial genes and their transfer to the nucleus that may end in the total loss of the organelle chromosome. In this way, the initial single symbiotic event may have resulted in a large variability of types of mitochondria and cell nuclei carrying mtDNA fragments variable in number, size, and location.

\section{NUMTs, genes, and pseudogenes. Identification and variability}

Mitochondrial DNA sequencing reveals that mitochondria carry an incomplete set of genes for their own function, together to non-coding regions necessary for the regulation of mtDNA replication and expression. One remarkable example is the nuclear-encoded DNA polymerase gamma (PolG) that replicates and repairs mtDNA and is homologous to E. coli pol I (Ito and Braithwaite 1991). Polg nuclear mutations affect the maintenance and proofreading function of mtDNA (Bailey et al. 2009). Polg mice mutants suffer premature ageing and various deleterious effects (Trifunovic et al. 2004). This provides evidence that functional genes have been transferred from the mtDNA to the nDNA. Most of the major protein complexes involved in oxidative phosphorylation contains both nuclear- and mitochondrial-encoded subunits, which promotes interesting questions on the co-evolution of mitochondrial and nuclear genetic functional interactions within the same cell. Other mitochondrial genes transferred to nDNA are unable to be expressed, maybe due, among other reasons, to the different genetic codes used in the mitochondria and the cytosol. These genes reside in the nDNA as pseudogenes.

Lopez et al. (1994) coined the term NUMT (nuclear mitochondrial DNA segment) for mtDNA sequences present in eukaryotic nuclei, which is generally applied to pseudogenes only or, in broad sense, to mitochondrial sequences found in nDNA ignoring if they are expressed or not. Singh et al. (2017) used numtogenesis to refer to the transfer of mtDNA into the nuclear genome or, less specifically, the transfer of mitochondrial components into the nucleus. Similarly, in the case of plants, the term NUPT refers to chloroplast DNA (cpDNA) inserted in nDNA.

NUMT identification is important both for the annotation and understanding of the genomes and for evolutionary studies. The first works identifying NUMTs were carried out by DNA hybridization, reporting the presence of sequences homologous to mitochondrial genes in nDNA, mainly rRNA genes and cytochrome oxidase subunit I gene. For example, Fukuda et al. (1985) isolated phage clones carrying DNAs homologous to human mtDNA, estimating that human nDNA contains several hundred copies of mtDNA-like fragments. The variety of organisms where homology of mtDNA and cpDNA sequences was found in nDNA early made clear that this phenomenon was of general occurrence (Zhang and Hewitt 1996; Bensasson et al. 2001). The advances of whole genome sequencing projects allowed much more accurate studies, particularly in the case of the human 
genome. Mourier et al. (2001) presented the first extensive NUMT analysis; they found long NUMTs representing nearly all parts of the mtDNA. Woischnik and Moraes (2002) found 612 independent integrations of mitochondrial pseudogenes in the human genome evenly distributed among all nuclear chromosomes as well as within each individual chromosome.

The comparison of NUMTs among species shows a high variability. Richly and Leister (2004) compared 13 species with sequenced mitochondrial and nuclear genomes revealing large interspecific variation of NUMT copy number and size, although no clear explanation exists for the interspecific diversity of NUMTs and NUPTs (Leister 2005). In certain protists the low NUMT and NUPT number can be due to the low number of possible donor organelles per cell. Another possible explanation relates to interspecific differences in the efficiency of integration of organelle DNA into the nuclear genome.

Interestingly, a correlation between the abundance of NUMTs and the size of the nuclear or the mitochondrial genomes, or of the nuclear gene density, is not evident. However, in mosquitoes, Ding et al. (2018) carried out a NUMT analysis of 19 mosquito species and concluded that the number, total length, and density of NUMTs are significantly correlated with genome size; moreover NUMTs are an important cause of nuclear genome size expansion in mosquitoes. In fungi, Krampis et al. (2006) and Sacerdot et al. (2008) relied on a comparative analysis of the NUMT content. Results revealed large differences in NUMT number and organization across the species. In plants, Ko and Kim (2016) found that NUMT patterns vary from species to species.

NUMT variability is found intraspecifically or even intraindividually as well. Lough et al. $(2008,2015)$ studied NUMTs in a set of maize inbred lines, showing extensive NUMT variation in size and location among lines, suggesting that mtDNA is being incorporated or lost from the maize nuclear genome continuously. The same is true for cpDNA insertions; Roark et al. (2010) studied the NUPTs into maize chromosomes of the same lines. Like NUMTs, the positions of the NUPTs varied greatly among the lines, suggesting that the transfers are recent as well as frequent. In Aegilops speltoides, the distribution of organellar-derived insertions differed among populations (Ruban et al. 2014). Malik et al. (2016) found intraindividual variation of mtDNA levels that differed significantly in mouse tissues. This variability is not functionally irrelevant. Rand et al. (2006) compared the longevity in strains of Drosophila simulans carrying mtDNAs with varying levels of divergence. The interspecific mtDNA strains showed a very significant epistatic interaction effects depending of the nuclear and mtDNA origins.

The organellar DNA transferred to the nucleus can be deleted as demonstrated by Sheppard and Timmis (2009) using a kanamycin resistance gene (neo) transferred from cpDNA to the nucleus in tobacco. They found that the gene is highly unstable, with deletion often occurring within a single generation, indicating that plastid DNA insertion into and removal from the nuclear genome might be in dynamic equilibrium. Schneider et al. (2014) found reversible accumulation of mtDNA in the mouse nucleus. They studied the accumulation of mtDNA in embryonic and induced pluripotent stem cells, reporting that upon differentiation, the level of mtDNA in these nuclei was substantially reduced.

In spite of being essential for understanding the NUMT inheritance, there are few studies comparing the mtDNA between somatic and germ lines. Sato et al. (2007) compared the proportions of mitochondria carrying deleted mtDNA in various tissues at various ages. Certain somatic tissues showed increases in the proportion of deleted mtDNA with age, but the germ cells of females and their offspring showed a strong decrease in deleted mtDNA with maternal age. It seems that female germ cells have machinery that prevents the inheritance of defective mtDNA. De Paula et al. (2013b) have shown that female gametes of Aurelia aurita do not transcribe mtDNA, lack electron transport, and produce no free radicals. In contrast, male gametes actively transcribe mitochondrial genes for respiratory chain components and produce reactive oxygen species (ROS). The authors predict that quiescent oocyte mitochondria contain DNA as an unexpressed template that avoids mutation accumulation by being transmitted through the female germ line.

\section{NUMT generation and insertion mechanisms}

The existence of NUMTs implies that fragments of mtDNA must be produced in the mitochondria and reach the nucleus despite of the physical barriers it must overcome. It is generally accepted that mtDNA fragments are produced by the constant generation of ROS in the mitochondria (Barja 2013). The mtDNA, devoid of histones and located in the inner membrane of the mitochondria, very close to the place of ROS production, is more vulnerable than nDNA to oxidative damage caused by ROS.

Several mechanisms have been proposed that would facilitate the exit of mtDNA from the mitochondria and the entry into the nucleus. The most commonly accepted one is that the fragments generated by ROS in the mitochondria appear with a high 8-oxo-deoxyguanosine content in the cytoplasm due to alterations of the organelle membrane, either during division, fission/fusion events (Dimmer and Scorrano 2006), lysis (Mota 1963), mitophagy (Higgins and Coughlan 2014), or by the opening induction of a permeability transition pore (Patrushev et al. 2004; Garcia and Chavez 2007). Once in the cytoplasm, mtDNA is protected from nucleases thanks to a mechanism mediated by vacuoles, which are degraded when they contact the nucleus (Campbell and Thorsness 
1998). It has also been proposed (Kutsyi et al. 2005) that mtDNA could form a complex with DNA-binding histonelike proteins avoiding degradation. Other pathways enabling mtDNA to reach the nucleus are direct physical association between the mitochondrial and nuclear membranes (Mota 1963) or encapsulation of the mitochondria inside the nucleus (Jensen et al. 1976). It has been suggested that mtDNA could be transferred to the nucleus in the form of mRNA with the help of a reverse transcriptase to complete the process (Rodley et al. 2012). Nevertheless, some experimental studies do not support this possibility. For example, Woischnik and Moraes (2002) found that large NUMTs found in nDNA contain two or more mitochondrial genes with fragments of noncoding regions. As well, Falkenberg et al. (2007) reported that the mitochondrial transcripts are significantly shorter than the sequences of many NUMTs.

The mtDNA fragments that enter the nucleus must interact with the nDNA before their integration for NUMTs formation. All regions of the mitochondrial genome are able to interact with nDNA, as evidenced by the fact that recent human NUMTs contain fragments originated from the entire mtDNA (Dayama et al. 2014). However, Doynova et al. (2016), using chromosome conformation capture techniques to detect physical interactions between mt- and nDNA in mammalian cells, showed that the D-loop region exhibited a higher tendency to interact with the nuclear genome, probably because this region is more prone to breakage than other mtDNA regions (Rothfuss et al. 2010).

MtDNA fragments are inserted into the nucleus during the process of double-strand breaks (DSBs) repair via the non-homologous end-joining (NHEJ) machinery. This hypothesis was first proposed by Blanchard and Schmidt (1996) and later confirmed by Ricchetti et al. (1999) in a study on yeast in experimental conditions where homologous recombination, the other DSBs repair mechanism, was avoided. Similar results revealing the involvement of the NHEJ mechanism in the integration of mtDNA in the nucleus were obtained in humans (Ricchetti et al. 2004). Hazkani-Covo and Covo (2008) identified 35 and 55 lineage-specific NUMTs in the human and chimpanzee genomes, respectively, showing that in $54 \%$ of the NUMT integration events examined no deletions were detected. Considering that DSBs repair without NUMTs requires nuclease processing of DNA ends that usually produce small deletions, these results led the authors to propose that mtDNA fragments provide an alternative to nuclease activity in DSBs repair via NHEJ using the mtDNA as a filler DNA.

Mitochondrial fragments are transferred to the nucleus in a single or several copies and, in some cases, mtDNA suffers rearrangements prior or during the insertional event (Ricchetti et al. 1999; Huang et al. 2003). These rearrangements include tandem duplications or changes in gene order with respect to the organelle or- ganization, as well as the presence of fragments belonging to different regions of the mitochondrial genome. Nevertheless, the evidence that older NUMTs tend to be shorter than recent ones (Bensasson et al. 2003) and large recent insertions usually correspond to the whole sequence of the mitochondrial genome (Huang et al. 2005) indicates that NUMTs can be fragmented after insertion. Matsuo et al. (2005) and Michalovova et al. (2013) demonstrated that, once inside the nuclear genome, organellar DNA exhibits insertion instability, with fragmentation and recombinational events in most cases mediated by nuclear mobile elements.

\section{mtDNA nuclear insertions are involved in disease and ageing}

Certain de novo mtDNA fragment insertions may produce harmful effects, being involved in human disease. Willett-Brozick et al. (2001) described for the first time a spontaneous recent germ line insertion of human mtDNA at the breakpoint junctions of a familial constitutional reciprocal translocation. The 41-bp mitochondrial fragment was captured during the repair of the DSBs involved in the translocation, revealing the implication of this mechanism in the mtDNA transfer to the nucleus. A 251-bp mtDNA insertion was found in a patient suffering from severe plasma factor VII deficiency, a rare bleeding disorder. The mitochondrial fragment, containing the encoding DNA for tRNA-Phe and part of the 12S subunit of rRNA, was integrated in the IVS 4 acceptor splice site (Borensztajn et al. 2002). Turner et al. (2003) described a rare case of Pallister-Hall syndrome caused by a transfer of mtDNA to the nuclear genome. The fragment, 72-bp long, was found in exon 14 of the GLI3 gene, generating a premature stop codon and producing a truncated protein. Interestingly, this case was associated with the radioactive contamination that followed the Chernobyl accident, revealing again the link between NUMTs and DNA repair and instability. Cases of human disease, type IV mucolipidosis (Goldin et al. 2004) and Usher syndrome type IC (Ahmed et al. 2002), have also been related to mtDNA fragment nuclear insertions. Millar et al (2010) reported an isolated case of lissencephaly caused by the insertion of a mitochondrial genomederived DNA sequence into the 50 untranslated region of the PAFAH1B1 (LIS1) gene.

The mtDNA fragments are produced, transported, and inserted from the mitochondria towards the nucleus during the lifetime of the individuals in vital mitotic and post-mitotic tissues. The consequences for the individual itself are important. Richter (1988) first proposed that the oxidatively generated mtDNA fragments that escaped from mitochondria and become integrated into the nuclear genome might transform cells to a cancerous state.

Several studies have concluded that both insertion and change in copy number of mtDNA fragments are associated with carcinogenesis because mtDNA insertion 
may disrupt tumor suppressor genes or activate oncogenes, contributing to cancer development. Remarkably, surveys of thousands of human whole-cancer genomes have shown that chromosomal rearrangements are frequently combined with mtDNA fragments somatically transferred to the nucleus (Ju et al. 2015; Ju 2016). Mitochondrial fragments have been identified in the $c$-myc oncogene of HeLa cells (Shay et al. 1991). Srinivasainagendra et al. (2017) reported increased mtDNA insertions in the nuclear genomes of colorectal adenocarcinomas. Mobile LINE elements with mitochondrial inserts were found in the nuclear genome of mouse and rat tumors (Hadler et al. 1998).

The genomic instability caused by the insertions of mtDNA in the nuclear genome during individual lifetime has also implications in ageing. The mitochondrial free radical theory of ageing (MFRTA) (Harman 1972) proposes that ageing is caused by the cumulative damage produced by the constant generation of ROS in the mitochondria throughout the life of the individuals. It has been found that the mtDNA fragments have a higher level of 8-oxo-deoxyguanosine, a marker of ROS-induced DNA oxidative damage, than wild type non-fragmented mtDNA (Suter and Richter 1999), supporting ROS as causing agents of producing these fragments. The 8-oxodG levels are lower in the heart and brain of long-lived than short-lived animal species in the case of mtDNA and not in the nDNA (Barja and Herrero 2000). MFRTA was updated and reviewed by Barja (2013, 2017, 2019).

Studies in rat have shown that the amount of mtDNA in the nucleus increases with the age of individuals in liver and brain (Caro et al. 2010). The mitochondrial regions identified corresponded to cytochrome oxidase III and $16 \mathrm{~S}$ rRNA. Interestingly, these fragments contained the same SNPs found in the mitochondrial genome of the same individuals, revealing their recent origin. Similar results showing an association between increasing the copy number of mtDNA insertions and ageing were observed in yeast, where the insertion of mtDNA fragments decreases the chronological life span, measured as the time that non-proliferative cell populations can survive (Cheng and Ivessa 2010, 2012). Likewise, it has been proven in mice (Martínez-Cisuelo et al. 2016) that agerelated mtDNA fragment insertion is associated with the production of ROS in the mitochondria throughout the life of individuals. The amount of mtDNA found in the nuclei of hepatocytes and the rate of mitROS production of old mice decreased to levels similar to those found in young individuals (100\% reversion) when the old ones were treated during 7 weeks with rapamycin. This drug, which inhibits the TOR (Target Of Rapamycin) protein, decreases the production of ROS in the mitochondrial complex I, and it is the only one described to date capable of increasing longevity in mammals in a reproducible way (Harrison et al. 2009).

\section{NUMTS are frequently located at pericentromeric and (or) subtelomeric regions}

In situ localization of NUMTs to chromosomes using mtDNA as probe provides data of particular interest because NUMT position at specific chromosomes or chromosome regions may reveal important features; however, not many works include these studies. In situ localization in animal and plant species reveals frequent interactions between mtDNA and nuclear heterochromatin such as the pericentromeric and (or) subtelomeric regions.

Vaughan et al. (1999) localized mtDNA sequences to meiotic chromosomes of several orthopteran species using in situ hybridization; mtDNA localization varied between species being centromeric, telomeric, or present throughout the chromosomes in different species. Stupar et al. (2001) found an insertion of 270-kb mtDNA into the pericentric region on the short arm of chromosome 2 of Arabidopsis. Michalovova et al. (2013) found mtDNA and cpDNA insertions located at the pericentromeric regions of Arabidopsis and rice. Lough et al. $(2008,2015)$ studied mtDNA insertions in a set of maize inbred lines and found extensive variation in size and location among lines, but in many lines the signals were located near the centromere or the telomere. Caro et al. (2010) using FISH in bone marrow cells of young and old rats reported that 10 pairs of chromosomes showed mtDNA signal located always at the pericentromeric region in rats of both ages. Similar results were observed in the telocentric chromosomes of mice where mtDNA fragments colocalize with pericentromeric satellite sequences (Martinez-Cisuelo et al. 2016). Mustafa et al. (2018) obtained near-complete genomes of mitochondria from wild sheep species or subspecies. In situ localization showed strong hybridization to the centromeric regions of all autosomal sheep chromosomes, but not the $\mathrm{Y}$, with varying abundance of different mitochondrial regions. Recently, Koo et al. (2018) have developed a technique of single-molecule mtFIBER FISH to study numtogenesis in human, to aid in establishing a role for numtogenesis in cancer and other human diseases.

$\mathrm{B}$ chromosomes (Bs) are supernumerary to the normal chromosome set (A chromosomes or As) that are not required for the normal growth and development of the B-carrier organism. In situ localization of mtDNA and cpDNA has been carried out in the Bs of rye and Aegilops speltoides (Martis et al. 2012; Ruban et al. 2014). In both species, the B has accumulated large and significantly greater amounts of cp- and mtDNA-derived sequences than the chromosomes of the normal set. Almost all parts of the chloroplast and mitochondrial genomes were found on the Bs. In rye, the organellar DNA localizes in pericentromeric regions, whereas in Ae. speltoides insertions were found along both arms of the Bs except at the pericentromere.

According to Michalovova et al. (2013), the frequent finding of mtDNA fragments located at the pericentro- 
meric regions is explained because the centromeres constitute a stable genomic environment, being regions poor in genes, and because frequent DSBs occur that are repaired using the NHEJ mechanism, thanks to which the fragments are incorporated into the nuclear genome (Matsuo et al. 2005). This model predicts that in species with great genome dynamism these blocks of pericentromeric mtDNA would be fragmented by the insertion of transposable elements (TEs) taking them to other regions of the genome, away from the centromeres. In addition, the ectopic recombination mediated by TEs would be responsible for the remodeling of the regions where the mtDNA fragments are found, producing their exit from the genome either when they are forming a pericentromeric block or when they have been fragmented and are found in other locations.

Centromeric function is highly conserved in all eukaryotes; however, centromeric DNA shows remarkable sequence variability between species or even among different chromosomes of the same species, as for example between As and Bs (Jones et al. 2008). This makes it remarkably difficult to define and identify the DNA elements responsible for centromere activity. This large divergence is possible thanks to the coevolution between the centromeric sequences and the kinetochore proteins. All centromeres consist in repeated sequences, making difficult its sequencing. The functional domain of the centromere consists in satellites, centromeric retrotransposons, or both. This functional region is surrounded by repeated sequences, possibly including mtDNA insertions in many cases, which are important for other centromeric functions such as chromatid cohesion. The role of each of the regions is important in the processes of cohesion and dissociation that occur in the centromeres during chromosomal segregation in cell division (Guenatri et al. 2004).

\section{mtDNA insertions may be considered as parasitic genetic elements}

Parasitic genetic elements (called selfish genetic elements as well) are those that produce harmful effects to the host organisms, whereas they are maintained in populations because they undergo a mechanism of drive resulting in higher than Mendelian transmission. One of the best-studied examples of parasitic genetic elements are the Bs, supernumerary to the normal As set, where population dynamics of B-carrying species depends on the strength of non-Mendelian drive mechanisms counteracted by the harmful effect of Bs on the fitness of B-carrier individuals (Jones et al. 2008).

TEs are considered parasitic elements as well, because they may produce harmful effects when they move producing deleterious mutations and possess mechanisms that promote their own higher than Mendelian transmission. Besides their parasitic features, the TEs have been demonstrated to be a main evolutionary force influenc- ing or even driving the genomic and karyotypic evolution (Ayarpadikannan and Kim 2014).

Interestingly, NUMTs and TEs share certain common features: they are "passengers" of the nucleus that play various roles in processes affecting genome evolution and genetic instability. They are instable per se and produce genomic instability. The effects of TE and NUMTs in the genome vary from negligible to harmful, producing various genetic disorders and cancer. Recently, the possible adaptive phenotypic changes associated with TEs were considered (Schrader and Schmitz 2019), indicating that the activity of TEs might facilitate adaptive responses to environmental challenges.

Mitochondrial ROS production breaks mtDNA into mtDNA fragments that are transferred to the nucleus, mainly at pericentromeric regions, during the lifetime of the individual, promoting ageing at the nuclear level. The mechanisms involved potentially include harmful effects such as the induction of major chromosomal rearrangements, inhibition of cell division and transposonmediated insertion, and modification of regulatory regions and structural coding genes (Barja 2017). On the other hand, the generation of NUMTs seems to be a nonstop process overpassing the Mendelian transmission. These parasitic properties ensure their survival and spread in natural populations, even against the harmful effects on the host. The accumulation of mtDNA fragments mainly at centromeric and also subtelomeric regions is important to understand the transmission and integration of NUMTs into the genomes which might promote their own higher than Mendelian transmission.

The potential harmful effect of NUMTs is evidenced in the case of the parasitic Bs of rye and Ae. speltoides. The Bs show a much higher amount of organelle-derived DNA than the As of the normal set. Insertions into As may disrupt gene function with lethal consequences. In contrast the Bs, which are not required for growth and development, can tolerate more mutations (Martis et al. 2012; Ruban et al. 2014).

As in the case of TEs, the proposed parasitic features of NUMTs does not mean that they are not important in cell evolution; contrarily, as stated by Leister (2005) NUMTs and NUPTs are more than only "mutagens". NUMTs and NUPTs can influence nuclear processes such as replication or transcription, or they might even rebuild genes and their products by providing new exons. If they can, this would constitute, in addition to the ancient transfer of entire prokaryotic genes to the nucleus, a further contribution that organelles make to the evolution of nuclear genomes. Popadin et al. (2017) found that the pseudogene Ps5, a large 9Kb-NUMT, was independently fixed in populations of gorilla and the human/chip nascent populations, which implies that the spread of the pseudogene within and across populations might have been driven by positive selection. Besides, the rate of NUMT insertion is not constant and may correlate with 
critical points in evolution. Gunbin et al. (20017a, 2017b) estimated the times of incorporation of 18 selected NUMTs during the period of evolution of the human lineage after separating from the chimpanzee and found a non-random rate of insertion, with one cluster situated around 2.8 million years ago, corresponding to a period of major climate change and the time of emerge of the genus Homo. Similarly, the reconstruction of the NUMT insertion history in two bird species, Geospiza fortis and Zonotrichia albicollis, and their closed relatives, showed a remarkable acceleration of insertions in the ancestor of both species followed by a slower accumulation in each lineage (Liang et al. 2018). The results indicate that mtDNA insertions represent a major source of nuclear chromosomal variation. Whether or not mtDNA insertions might play an adaptive role in the speciation processes is a matter of future research.

In the case of mtDNA fragment insertion into nDNA, the co-evolution between nuclear and cytoplasmic genomes has to be considered differently in unicellular or asexually-reproducing versus multi-cellular organisms with separated somatic and germ lines. In asexuallyreproducing organisms, the NUMTs inserted into nDNA will be transmitted to the progeny, unless a hypothetical detachment mechanism were present; whereas in organisms with separated somatic and germ lines, only those NUMTS present in the gamete DNA will be transmitted. The great majority of NUMT studies were carried out in somatic tissues, thus lacking much information on this main point.

It should be also considered that the harmful effect of NUMT insertion during the lifetime of individuals (cancer, ageing) usually occurs after the reproduction of the individual has occurred, and therefore it is reasonable to think that they will not affect significantly the fertility either at the individual or population levels. It was also hypothesized (Richly and Leister 2004) that factors, such as the number and (or) stability of mitochondria in the germline, or species-specific mechanisms controlling accumulation/loss of nDNA, might be responsible for the interspecific diversity in NUMT accumulation, but as far as we know there are no population quantitative studies relating the NUMT number and size polymorphism with effects on fitness.

Various studies propose a different role of female and male germ lines for NUMT transmission. It has been hypothesized (Woischnik and Moraes 2002) that sperm mtDNA, which is released from degenerating mitochondria after fertilization, could be an important source of nuclear mtDNA pseudogenes transmitted to the progeny. In contrast, Sato et al. (2007) proposed that female germ cells have machinery that prevents the inheritance of defective mtDNA to the following generation because germ cells are kept for a long time until they are ovulated. De Paula et al. (2013a, 2013b) indicated that suppressed mitochondrial metabolism in the female germ line may constitute a mechanism for increasing the fidelity of mtDNA inheritance. They proposed that quiescent oocyte mitochondria contain DNA as an unexpressed template that avoids mutational accumulation by being transmitted through the female germ line. The avoidance of ROS-dependent mutation would be the evolutionary pressure underlying maternal mitochondrial inheritance and the developmental origin of the female germ line.

Female meiosis, and megagametogenesis in the case of plants, are moments of the vital cycle where there is opportunity for a non-Mendelian drive mechanism to occur because only one of the four meiotic products (and one of the nuclei of the megaspores) acts as female gamete. When competition occurs for a meiotic product to be included in the female gamete, the process is called female meiotic drive, which is mainly associated to repetitive sequences, such as those present in centromeres, subtelomeric heterochromatin, and other chromosome regions mainly composed by repetitive sequences (Puertas and Villasante 2013; Lindholm et al. 2016). To explain centromere drive, Iwata-Otsubo et al. (2017) proposed that amplified repetitive sequences act as parasitic elements by promoting expansion of CENP-A chromatin and increased transmission through the female germline. The study of female meiotic drive for mtDNA insertions remains to be studied.

\section{References}

Ahmed, Z.M., Smith, T.N., Riazuddin, S., Makishima, T., Ghosh, M., Bokhari, S., et al. 2002. Nonsyndromic recessive deafness DFNB18 and Usher syndrome type IC are allelic mutations of USHIC. Hum Genet. 110(6): 527-531. doi:10.1007| s00439-002-0732-4. PMID:12107438.

Andersson, S.G., Zomorodipour, A., Andersson, J.O., Sicheritz-Pontén, T., Alsmar, U.C., Podowski, R.M., et al. 1998. The genome sequence of Rickettsia prowazekii and the origin of mitochondria. Nature, 396(6707): 133-140. doi:10.1038/24094. PMID:9823893.

Ayarpadikannan, S., and Kim, H.S. 2014. The impact of transposable elements in genome evolution and genetic instability and their implications in various diseases. Genomics Inform. 12(3): 98-104. doi:10.5808/GI.2014.12.3.98. PMID:25317108.

Bailey, L.J., Cluett, T.J., Reyes, A., Prolla, T.A., Poulton, J., Leeuwenburgh, C., and Holt, I.J. 2009. Mice expressing an error-prone DNA polymerase in mitochondria display elevated replication pausing and chromosomal breakage at fragile sites of mitochondrial DNA. Nucleic Acids Res. 37(7): 2327-2335. doi:10.1093/nar/gkp091. PMID:19244310.

Barja, G. 2013. Updating the mitochondrial free radical theory of aging: an integrated view, key aspects, and confounding concepts. Antioxid. Redox Signal. 19(12): 1420-1445. doi:10. 1089/ars.2012.5148. PMID:23642158.

Barja, G. 2017. The Cell Aging Regulation System (CARS). React. Oxygen Species, 3(9): 148-183. doi:10.20455/ros.2017.829.

Barja, G. 2019. Towards a unified mechanistic theory of aging. Exp. Gerontol. 124: 110627. doi:10.1016/j.exger.2019.05.016. PMID:31173843.

Barja, G., and Herrero, A. 2000. Oxidative damage to mitochondrial DNA is inversely related to maximum life span in the heart and brain of mammals. FASEB J. 14: 312-318. doi:10. 1096/fasebj.14.2.312. PMID:10657987. 
Bensasson, D., Zhang, D., Hartl, D.L., and Hewitt, G.M. 2001. Mitochondrial pseudogenes: Evolution's misplaced witnesses. Trends Ecol. Evol. 16: 314-321. doi:10.1016/S0169-5347(01)021516. PMID:11369110.

Bensasson, D., Feldman, M.W., and Petrov, D.A. 2003. Rates of DNA duplication and mitochondrial DNA insertion in the human genome. J. Mol. Evol. 57: 343-354. doi:10.1007/s00239003-2485-7. PMID:14629044.

Blanchard, J.L., and Schmidt, G.W. 1996. Mitochondrial DNA migration events in yeast and humans: integration by a common end-joining mechanism and alternative perspectives on nucleotide substitution patterns. Mol. Biol. Evol. 13(3): 537-548. doi:10.1093/oxfordjournals.molbev.a025614. PMID: 8742642

Borensztajn, K., Chafa, O., Alhenc-Gelas, M., Salha, S., Reghis, A., Fischer, A.M., and Tapon-Bretaudière, J. 2002. Characterization of two novel splice site mutations in human factor VII gene causing severe plasma factor VII deficiency and bleeding diathesis. Br. J. Haematol. 117: 168-171. doi:10.1046/j.1365-2141.2002.03397.x. PMID:11918550.

Burger, G., Gray, M.W., Forget, L., and Lang, B.F. 2013. Strikingly bacteria-like and gene-rich mitochondrial genomes throughout jakobid protists. Genome Biol. Evol. 5(2): 418-438. doi:10. 1093/gbe/evt008. PMID:23335123.

Campbell, C.L., and Thorsness, P.E. 1998. Escape of mitochondrial DNA to the nucleus in yme1 yeast is mediated by vacuolar-dependent turnover of abnormal mitochondrial compartments. J. Cell Sci. 111: 2455-2464. PMID:9683639.

Caro, P., Gomez, J., Arduini, A., González-Sánchez, M., González-García, M., Borras, C., et al. 2010. Mitochondrial DNA sequences are present inside nuclear DNA in rat tissues and increase with age. Mitochondrion, 10(5): 479-486. doi:10. 1016/j.mito.2010.05.004. PMID:20546951.

Cheng, X., and Ivessa, A.S. 2010. The migration of mitochondrial DNA fragments to the nucleus affects the chronological aging process of Saccharomyces cerevisiae. Aging Cell, 9: 919-923. doi:10.1111/j.1474-9726.2010.00607.x. PMID:20626726.

Cheng, X., and Ivessa, A.S. 2012. Accumulation of linear mitochondrial DNA fragments in the nucleus shortens the chronological life span of yeast. Eur. J. Cell Biol. 91: 782-788. doi:10.1016/j.ejcb.2012.06.005. PMID:22857949.

Dayama, G., Emery, S.B., Kidd, J.M., and Mills, R.E. 2014. The genomic landscape of polymorphic human nuclear mitochondrial insertions. Nucleic Acids Res. 42(20): 12640-12649. doi:10.1093/nar/gku1038. PMID:25348406.

de Paula, W.B., Agip, A.N., Missirlis, F., Ashworth, R., Vizcay-Barrena, G., Lucas, C.H., and Allen, J.F. 2013a. Female and male gamete mitochondria are distinct and complementary in transcription, structure, and genome function. Genome Biol. Evol. 5(10): 1969-1977. doi:10.1093/gbe/evt147. PMID: 24068653.

de Paula, W.B., Lucas, C.H., Agip, A.N., Vizcay-Barrena, G., and Allen, J.F. 2013b. Energy, ageing, fidelity and sex: oocyte mitochondrial DNA as a protected genetic template. Philos. Trans. R. Soc. B Biol. Sci. 368(1622): 20120263. doi:10.1098/rstb. 2012.0263. PMID:23754815.

Dimmer, K.S., and Scorrano, L. 2006. (De)constructing mitochondria: what for? Physiology, 21: 233-241. doi:10.1152/physiol. 00010.2006. PMID:16868312.

Ding, Y.R., Li, B., Zhang, Y.J., Mao, Q.M., and Chen, B. 2018. Complete mitogenome of Anopheles sinensis and mitochondrial insertion segments in the nuclear genomes of 19 mosquito species. PLoS ONE, 13(9): e0204667. doi:10.1371/journal. pone.0204667. PMID:30261042.

Doynova, M.D., Berretta, A., Jones, M.B., Jasoni, C.L., Vickers, M.H., and O'Sullivan, J.M. 2016. Interactions between mitochondrial and nuclear DNA in mammalian cells are non-random. Mitochondrion, 30: 187-196. doi:10.1016/j. mito.2016.08.003. PMID:27503698.

Embley, T.M., van der Giezen, M., Horner, D.S., Dyal, P.L., and Foster, P. 2003. Mitochondria and hydrogenosomes are two forms of the same fundamental organelle. Philos. Trans. R. Soc. B Biol. Sci. 358(1429): 191-202. doi:10.1098/rstb.2002.1190. PMID:12594927.

Falkenberg, M., Larsson, N.G., and Gustafsson, C.M. 2007. DNA replication and transcription in mammalian mitochondria. Annu. Rev. Biochem. 76: 679-699. doi:10.1146/annurev.biochem. 76.060305.152028. PMID:17408359.

Fukuda, M., Wakasugi, S., Tsuzuki, T., Nomiyama, H., Shimada, K., and Miyata, T. 1985. Mitochondrial DNA-like sequences in the human nuclear genome. Characterization and implications in the evolution of mitochondrial DNA. J. Mol. Biol. 186: 257-266. doi:10.1016/0022-2836(85)90102-0. PMID:3003363.

Garcia, N., and Chavez, E. 2007. Mitochondrial DNA fragments released through the permeability transition pore correspond to specific gene size. Life Sci. 81: 1160-1166. doi:10.1016/ j.lfs.2007.08.019. PMID:17870132.

Goldin, E., Stahl, S., Cooney, A.M., Kaneski, C.R., Gupta, S., Brady, R.O., et al. 2004. Transfer of a mitochondrial DNA fragment to MCOLN1 causes an inherited case of mucolipidosis IV. Hum. Mutat. 24: 460-465. doi:10.1002/humu.20094. PMID:15523648.

Guenatri, M., Bailly, D., Maison, C., and Almouzni, G. 2004. Mouse centric and pericentric satellite repeats form distinct functional heterochromatin. J. Cell Biol. 166(4): 493-505. doi: 10.1083/jcb.200403109. PMID:15302854.

Gunbin, K., Peshkin, L., Popadin, K., Annis, S., Ackermann, R.R., and Khrapko, K. 2017a. Integration of mtDNA pseudogenes into the nuclear genome coincides with speciation of the human genus. A hypothesis. Mitochondrion, 34: 20-23. doi: 10.1016/j.mito.2016.12.001. PMID:27979772.

Gunbin, K., Peshkin, L., Popadin, K., Annis, S., Ackermann, R.R., and Khrapko, K. 2017b. Data on the time of integration of the human mitochondrial pseudogenes (NUMTs) into the nuclear genome. Data in Brief, 13: 536-544. doi:10.1016/j.dib. 2017.05.024. PMID:28702491.

Hadler, H.I., Devadas, K., and Mahalingam, R. 1998. Selected nuclear LINE elements with mitochondrial-DNA-like inserts are more plentiful and mobile in tumor than in normal tissue of mouse and rat. J. Cell. Biochem. 68(1): 100-109. doi:10. 1002/(SICI)1097-4644(19980101)68:1<100::AID-JCB10>3.0.CO;2-L. PMID:9407318.

Harman, M.D. 1972. The biologic clock: the mitochondria? J. Am. Geriatr. Soc. 20: 145-147. doi:10.1111/j.1532-5415.1972. tb00787.x. PMID:5016631.

Harrison, D.E., Strong, R., Sharp, Z.D., Nelson, J.F., Astle, C.M., Flurkey, K., et al. 2009. Rapamycin fed late in life extends lifespan in genetically heterogeneous mice. Nature, 460(7253): 392-395. doi:10.1038/nature08221. PMID:19587680.

Hazkani-Covo, E., and Covo, S. 2008. Numt-mediated doublestrand break repair mitigates deletions during primate genome evolution. PLoS Genet. 4: e1000237. doi:10.1371/journal. pgen.1000237. PMID:18949041.

Higgins, G.C., and Coughlan, M.T. 2014. Mitochondrial dysfunction and mitophagy: the beginning and end to diabetic nephropathy? Br.J. Pharmacol.171(8):1917-1942. doi:10.1111/bph. 12503. PMID:24720258.

Huang, C.Y., Ayliffe, M.A., and Timmis, J.N. 2003. Direct measurement of the transfer rate of chloroplast DNA into the nucleus. Nature, 422: 72-76. doi:10.1038/nature01435. PMID: 12594458.

Huang, C.Y., Grünheit, N., Ahmadinejad, N., Timmis, J.N., and Martin, W. 2005. Mutational decay and age of chloroplast and mitochondrial genomes transferred recently to angiosperm 
nuclear chromosomes. Plant Physiol. 138(3): 1723-1733. doi: 10.1104/pp.105.060327. PMID:15951485.

Ito, J., and Braithwaite, D.K. 1991. Compilation and alignment of DNA polymerase sequences. Nucleic Acids Res. 19(15): 40454057. doi:10.1093/nar/19.15.4045. PMID:1870963.

Iwata-Otsubo, A., Dawicki-McKenna, J.M., Akera, T., Falk, S.J., Chmátal, L., Yang, K., et al. 2017. Expanded Satellite repeats amplify a discrete CENP-A nucleosome assembly site on chromosomes that drive in female meiosis. Curr. Biol. 27: 23652373.e8. doi:10.1016/j.cub.2017.06.069. PMID:28756949.

Jensen, H., Engedal, H., and Saetersdal, T.S. 1976. Ultrastructure of mitochondria-containing nuclei in human myocardial cells. Virchows Arch. B Cell Pathol. 21: 1-12. doi:10.1007| BF02899139.

Jones, R.N., González-Sánchez, M., González-García, M., Vega, J.M., and Puertas, M.J. 2008. Chromosomes with a life of their own. Cytogenet. Genome Res. 120(3-4): 265-280. doi:10. 1159/000121076. PMID:18504356.

Ju, Y.S. 2016. Intracellular mitochondrial DNA transfers to the nucleus in human cancer cells. Curr. Opin. Genet. Dev. 38: 23-30. doi:10.1016/j.gde.2016.02.005. PMID:27010587.

Ju, Y.S., Tubio, J.M., Mifsud, W., Fu, B., Davies, H.R., Ramakrishna, M., et al. 2015. Frequent somatic transfer of mitochondrial DNA into the nuclear genome of human cancer cells. Genome Res. 25(6): 814-824. doi:10.1101/gr.190470. 115. PMID:25963125.

Ko, Y.J., and Kim, S. 2016. Analysis of Nuclear Mitochondrial DNA Segments of nine plant species: size, distribution, and insertion loci. Genomics Inform. 14: 90-95. doi:10.5808/GI. 2016.14.3.90. PMID:27729838.

Koo, D.H., Singh, B., Jiang, J., Friebe, B., Gill, B.S., Chastain, P.D., et al. 2018. Single molecule mtDNA fiber FISH for analyzing numtogenesis. Anal. Biochem. 552: 45-49. doi:10.1016/j.ab. 2017.03.015. PMID:28322800.

Krampis, K., Tyler, B.M., and Boore, J.L. 2006. Extensive variation in nuclear mitochondrial DNA content between the genomes of Phytophthora sojae and Phytophthora ramorum. Mol. Plant Microbe Interact. 19(12): 1329-1336. doi:10.1094/MPMI19-1329. PMID:17153917.

Kutsyi, M.P., Gouliaeva, N.A., Kuznetsova, E.A., and Gaziev, A.I. 2005. DNA binding proteins of mammalian mitochondria. Mitochondrion, 5: 35-44. doi:10.1016/j.mito.2004.09.002. PMID: 16060291.

Leister, D. 2005. Origin, evolution and genetic effects of nuclear insertions of organelle DNA. Trends Genet. 21(12): 655-663. doi:10.1016/j.tig.2005.09.004. PMID:16216380.

Liang, B., Wang, N., Li, N., Kimball, R.T., and Braun, E.L. 2018. Comparative genomics reveals a burst of homoplasy-free Numt insertions. Mol. Biol. Evol. 35(8): 2060-2064. doi:10.1093/ molbev/msy112. PMID:29860531.

Lindholm, A.K., Dyer, K.A., Firman, R.C., Fishman, L., Forstmeier, W., Holman, L., et al. 2016. The ecology and evolutionary dynamics of meiotic drive. Trends Ecol. Evol. 31(4): 315-326. doi:10.1016/j.tree.2016.02.001. PMID:26920473.

Lopez, J.V., Yuhki, N., Masuda, R., Modi, W., and O'Brien, S.J. 1994. Numt, a recent transfer and tandem amplification of mitochondrial DNA in the nuclear genome of the domestic cat. J. Mol. Evol. 39(2): 174-190. doi:10.1007/BF00163806. PMID:7932781.

Lough, A.N., Roark, L.M., Kato, A., Ream, T.S., Lamb, J.C., Birchler, J.A., and Newton, K.J. 2008. Mitochondrial DNA transfer to the nucleus generates extensive insertion site variation in maize. Genetics, 178(1): 47-55. doi:10.1534/genetics. 107.079624. PMID:18202357.

Lough, A.N., Faries, K.M., Koo, D.H., Hussain, A., Roark, L.M., Langewisch, T.L., et al. 2015. Cytogenetic and sequence analyses of mitochondrial DNA insertions in nuclear chromo- somes of maize. Genes Genomes Genetics (Bethesda), 5(11): 2229-2239. doi:10.1534/g3.115.020677. PMID:26333837.

Malik, A.N., Czajka, A., and Cunningham, P. 2016. Accurate quantification of mouse mitochondrial DNA without coamplification of nuclear mitochondrial insertion sequences. Mitochondrion, 29: 59-64. doi:10.1016/j.mito.2016.05.003. PMID: 27181048.

Martínez-Cisuelo, V., Gómez, J., García-Junceda, I., Naudí, A., Cabré, R., Mota-Martorell, N., et al. 2016. Rapamycin reverses age-related increases in mitochondrial ROS production at complex I, oxidative stress, accumulation of mtDNA fragments inside nuclear DNA, and lipofuscin level, and increases autophagy, in the liver of middle-aged mice. Exp. Gerontol. 83: 130-138. doi:10.1016/j.exger.2016.08.002. PMID: 27498120.

Martis, M.M., Klemme, S., Banaei-Moghaddam, A.M., Blattner, F.R., Macas, J., Schmutzer, T., et al. 2012. Selfish supernumerary chromosome reveals its origin as a mosaic of host genome and organellar sequences. Proc. Natl. Acad. Sci. U.S.A. 109(33): 13343-13346. doi:10.1073/pnas.1204237109. PMID:22847450.

Matsuo, M., Ito, Y., Yamauchi, R., and Obokata, J. 2005. The rice nuclear genome continuously integrates, shuffles, and eliminates the chloroplast genome to cause chloroplast-nuclear DNA flux. Plant Cell, 17: 665-675. doi:10.1105/tpc.104.027706. PMID:15705954.

Michalovova, M., Vyskot, B., and Kejnovsky, E. 2013. Analysis of plastid and mitocondrial DNA insertions in the nucleus (NUPTs and NUMTs) of six plant species: size, relative age and chromosomal localization. Heredity, 111(4): 314-320. doi:10. 1038/hdy.2013.51. PMID:23715017.

Millar, D.S., Tysoe, C., Lazarou, L.P., Pilz, D.T., Mohammed, S., Anderson, K., et al. 2010. An isolated case of lissencephaly caused by the insertion of a mitochondrial genome-derived DNA sequence into the $5^{\prime}$ untranslated region of the PAFAH1B1 (LIS1) gene. Hum. Genomics, 4(6): 384-393. doi:10. 1186/1479-7364-4-6-384. PMID:20846927.

Mota, M. 1963. Electron microscope study of relationship between nucleus and mitochondria in Chlorophytum capense (L.) Kuntze. Cytologia, 28: 409-416. doi:10.1508/cytologia.28.409.

Mourier, T., Hansen, A.J., Willerslev, E., and Arctander, P. 2001. The Human Genome Project reveals a continuous transfer of large mitochondrial fragments to the nucleus. Mol. Biol. Evol. 18(9): 1833-1837. doi:10.1093/oxfordjournals.molbev. a003971. PMID:11504863.

Mustafa, S.I., Schwarzacher, T., and Heslop-Harrison, J.S. 2018. Complete mitogenomes from Kurdistani sheep: abundant centromeric nuclear copies representing diverse ancestors. Mitochondrial DNA Part A, 29(8): 1180-1193. doi:10.1080/ 24701394.2018.1431226. PMID:29385875.

Patrushev, M., Kasymov, V., Patrusheva, V., Ushakova, T., Gogvadze, V., and Gaziev, A. 2004. Mitochondrial permeability transition triggers the release of mtDNA fragments. Cell. Mol. Life Sci. 61: 3100-3103. doi:10.1007/s00018-004-4424-1. PMID:15583871.

Popadin, K., Gunbin, K., Peshkin, L., Annis, S., Fleischmann, Z., Kraytsberg, Y., et al. 2017. Mitochondrial pseudogenes suggest repeated inter-species hybridization among direct human ancestors. bioRxiv. doi:10.1101/134502.

Puertas, M.J., and Villasante, A. 2013. Is the heterochromatin of meiotic neocentromeres a remnant of the early evolution of the primitive centromere? In Plant centromere biology. Chapter 8. Edited by J. Jiang and J.A. Birchler. Wiley \& Sons. pp. 95-109. doi:10.1002/9781118525715.ch8.

Rand, D.M., Fry, A., and Sheldahl, L. 2006. Nuclear-mitochondrial epistasis and drosophila aging: introgression of Drosophila simulans mtDNA modifies longevity in D. melanogaster nuclear backgrounds. Genetics, 172(1): 329-341. doi:10.1534/genetics. 105.046698. PMID:16219776. 
Ricchetti, M., Fairhead, C., and Dujon, B. 1999. Mitochondrial DNA repairs double strand breaks in yeast chromosomes. Nature, 402: 96-100. doi:10.1038/47076. PMID:10573425.

Ricchetti, M., Tekaia, F., and Dujon, B. 2004. Continued colonization of the human genome by mitochondrial DNA. PLoS Biol. 2: e273. doi:10.1371/journal.pbio.0020273. PMID:15361937.

Richly, E., and Leister, D. 2004. NUMTs in sequenced eukaryotic genomes. Mol. Biol. Evol. 21(6): 1081-1084. doi:10.1093/molbev/ msh110. PMID:15014143.

Richter, C. 1988. Do mitochondrial DNA fragments promote cancer and aging? FEBS Lett. 241: 1-5. doi:10.1016/00145793(88)81018-4. PMID:3197826.

Roark, L.M., Hui, A.Y., Donnelly, L., Birchler, J.A., and Newton, K.J. 2010. Recent and frequent insertions of chloroplast DNA into maize nuclear chromosomes. Cytogenet Genome Res. 129: 17-23. doi:10.1159/000312724. PMID:20628248.

Rodley, C.D., Grand, R.S., Gehlen, L.R., Greyling, G., Jones, M.B., and O'Sullivan, J.M. 2012. Mitochondrial-nuclear DNA interactions contribute to the regulation of nuclear transcript levels as part of the inter-organelle communication system. PLoS ONE, 7(1): e30943. doi:10.1371/journal.pone.0030943. PMID: 22292080.

Rothfuss, O., Gasser, T., and Patenge, N. 2010. Analysis of differential DNA damage in the mitochondrial genome employing a semi-long run real-time PCR approach. Nucleic Acids Res. 38(4). e24. doi:10.1093/nar/gkp1082. PMID:19966269.

Ruban, A., Fuchs, J., Marques, A., Schubert, V., Soloviev, A., Raskina, O., et al. 2014. B Chromosomes of Aegilops speltoides are enriched in organelle genome-derived sequences. PLoS ONE, 9(2): e90214. doi:10.1371/journal.pone.0090214. PMID:24587288.

Sacerdot, C., Casaregola, S., Lafontaine, I., Tekaia, F., Dujon, B., and Ozier-Kalogeropoulos, O. 2008. Promiscuous DNA in the nuclear genomes of hemiascomycetous yeasts. FEMS Yeast Res. 8(6): 846-857. doi:10.1111/j.1567-1364.2008.00409.x. PMID: 18673395.

Sato, A., Nakada, K., Shitara, H., Kasahara, A., Yonekawa, H., and Hayashi, J. 2007. Deletion-mutant mtDNA increases in somatic tissues but decreases in female germ cells with age. Genetics, 177(4): 2031-2037. doi:10.1534/genetics.107.081026. PMID:18073422.

Schneider, J.S., Cheng, X., Zhao, Q., Underbayev, C., Gonzalez, J.P., Raveche, E.S., et al. 2014. Reversible mitochondrial DNA accumulation in nuclei of pluripotent stem cells. Stem Cells Dev. 23(22): 2712-2719. doi:10.1089/scd.2013.0630. PMID:24964274.

Schrader, L., and Schmitz, J. 2019. The impact of transposable elements in adaptive evolution. Mol. Ecol. 28: 1537-1549. doi: 10.1111/mec.14794. PMID:30003608.

Shay, J.W., Baba, T., Zhan, Q.M., Kamimura, N., and Cuthbert, J.A. 1991. HeLa TG cells have mitochondrial DNA inserted into the c-myc oncogene. Oncogene, 6: 1869-1874. PMID:1923509.
Sheppard, A.E., and Timmis, J.N. 2009. Instability of plastid DNA in the nuclear genome. PLoS Genet. 5(1): e1000323. doi:10.1371/ journal.pgen.1000323. PMID:19119415.

Singh, K.K., Choudhury, A.R., and Tiwari, H.K. 2017. Numtogenesis as a mechanism for development of cancer. Semin. Cancer Biol. 47: 101-109. doi:10.1016/j.semcancer.2017.05.003. PMID:28511886.

Srinivasainagendra, V., Sandel, M.W., Singh, B., Sundaresan, A., Mooga, V.P., Bajpai, P., et al. 2017. Migration of mitochondrial DNA in the nuclear genome of colorectal adenocarcinoma. Genome Med. 9(1): 31. doi:10.1186/s13073-017-0420-6. PMID: 28356157.

Stupar, R.M., Lilly, J.W., Town, C.D., Cheng, Z., Kaul, S., Buell, C.R., and Jiang, J. 2001. Complex mtDNA constitutes an approximate $620-\mathrm{kb}$ insertion on Arabidopsis thaliana chromosome 2: Implication of potential sequencing errors caused by large-unit repeats. Proc. Natl. Acad. Sci. U.S.A. 98(9): 50995103. doi:10.1073/pnas.091110398. PMID:11309509.

Suter, M., and Richter, C. 1999. Fragmented mitochondrial DNA is the predominant carrier of oxidized DNA bases. Biochemistry, 38: 459-464. doi:10.1021/bi9811922. PMID:9890929.

Thrash, J.C., Boyd, A., Huggett, M.J., Grote, J., Carini, P., Yoder, R.J., et al. 2011. Phylogenomic evidence for a common ancestor of mitochondria and the SAR11 clade. Sci. Rep. 1: 13. doi:10.1038/srep00013. PMID:22355532.

Trifunovic, A., Wredenberg, A., Falkenberg, M., Spelbrink, J.N., Rovio, A.T., Bruder, C.E., et al. 2004. Premature ageing in mice expressing defective mitochondrial DNA polymerase. Nature, 429(6990): 417-423. doi:10.1038/nature02517. PMID: 15164064.

Turner, C., Killoran, C., Thomas, N.S., Rosenberg, M., Chuzhanova, N.A., Johnston, J., et al. 2003. Human genetic disease caused by de novo mitochondrial-nuclear DNA transfer. Hum Genet. 112: 303-309. doi:10.1007/s00439-002-0892-2. PMID:12545275.

Vaughan, H.E., Heslop-Harrison, J.S., and Hewitt, G.M. 1999. The localization of mitochondrial sequences to chromosomal DNA in orthopterans. Genome, 42(5): 874-880. doi:10.1139/ g99-020.

Willett-Brozick, J.E., Savul, S.A., Richey, L.E., and Baysal, B.E. 2001. Germ line insertion of mtDNA at the breakpoint junction of a reciprocal constitutional translocation. Hum. Genet. 109(2): 216-223. doi:10.1007/s004390100564. PMID:11511928.

Williams, B.A., Hirt, R.P., Lucocq, J.M., and Embley, T.M. 2002. A mitochondrial remnant in the microsporidian Trachipleistophora hominis. Nature, 418(6900): 865-869. doi:10.1038/nature00949. PMID:12192407.

Woischnik, M., and Moraes, C.T. 2002. Pattern of organization of human mitochondrial pseudogenes in the nuclear genome. Genome Res. 12: 885-893. doi:10.1101/gr.227202. PMID:12045142.

Zhang, D.X., and Hewitt, G.M. 1996. Nuclear integrations: challenges for mitochondrial DNA markers. Trends Ecol. Evol. 11: 247-251. doi:10.1016/0169-5347(96)10031-8. PMID:21237827. 